\title{
Thermally Activated Magnetisation Reversal at High Frequencies
}

\author{
Roy W Chantrell and Jonathan Hannay
}

Computational Magnetism Group. School of Electronic Engineering and Computer Systems, University of Wales Bangor, Dean Street, Bangor, LL57 1UT, UK

\begin{abstract}
A computational model of thermally activated magnetisation reversal in particulate materials is described. The model allows the study of the detailed relaxation behaviour of isolated (non-interacting) particles. Computed results show non-exponential relaxation over small energy barriers attributable to the absence of a unique relaxation time. The coercivity shows a rapid increase at very short measurement time which is important as regards ultimate switching speeds in recording. The effects of interparticle dipolar magnetic interactions are also introduced into the model. These have the effect of increasing the spread of relaxation times via the inevitable dispersion in the magnitude and direction of the local field.
\end{abstract}

Key Words: Thermal activation, Interaction Effects

\section{INTRODUCTION}

The time dependence of the magnetisation has been known since the pioneering work of Ewing [1] and Street and Wooley [2]. The later work characterised the time dependent magnetisation as a 'magnetic viscosity'. Magnetic viscosity is essentially an experimentally observable time-dependence of the magnetisation under steady external field conditions. For the past 10 years there has been an increasing interest in this phenomenon for fundamental and practical investigations of magnetic materials. The time dependence phenomenon arises from thermal activation over finite energy barriers. These energy barriers arise typically from an intrinsic anisotropy in granular materials or from the pinning mechanisms in materials whose magnetic behaviour is predominantly governed by domain wall motion.

Generally speaking, two approaches to micromagnetic simulations to magnetisation reversal have emerged. In most theoretical micromagnetic studies of hysteresis in recording media the assumption is made that magnetisation reversal occurs in the absence of any thermal perturbations. This standard micromagnetic approach evaluates the free energy of the system, comprising the anisotropy energy, the magnetostatic energy, the exchange energy and the energy of interaction with the applied field. This energy is minimised subject to the appropriate boundary conditions. This leads to an equilibrium state for the magnetisation. Magnetisation reversal occurs for some critical value of the applied field at which the local energy minimum vanishes. At this point an irreversible transition to a new stable state occurs. This approach has been very successful in predicting magnetisation reversal mechanisms in a wide variety of materials including longitudinal thin film media, particulate recording media and permanent magnets.

It is clear that the standard micromagnetic formalism is not capable of predicting the effects of thermal activation since this requires the determination of the energy barrier. A considerable amount of work on thermal activation has been carried out into the behaviour of the simplest case of a non-interacting spin relaxing in a potential provided by a uniaxial anisotropy energy and an applied magnetic field. In such a system it can be shown that relaxation is hindered by an energy barrier $E_{b}$ which can be calculated from the StonerWohlfarth theory [3].

For systems with relatively large energy barriers almost any formalism leads to an exponential relaxation of the magnetisation with a relaxation time given by the Arrhenius-Neel law [4]

$$
\tau^{-1}=f_{0} \exp \left(-E_{b} / k T\right)
$$

$f_{0}$ is a pre-exponential factor of the order $10^{9} \mathrm{~s}^{-1}$, and $E_{b}$ is the energy barrier to magnetisation reversal. Equation (1) represents a fairly crude approximation.

Firstly $f_{0}$ is not a constant and is in fact dependent on many important parameters such as the particle volume, the temperature and the external applied field. The precise form of the pre-exponential factor has been studied for example by Brown [5], Aharoni [6] and more recently by Coffey and coworkers [7]. Equally importantly the exponential form of the relaxation law is applicable only for relaxation over relatively large energy barriers and in fact equation (1) breaks down as $\mathrm{E}_{\mathrm{b}} / \mathrm{kT}$ approaches unity. The shortcomings of equation (1) are not apparent in studies of the so-called slow dynamic behaviour of magnetic materials characterised by a gradual change of the magnetisation over long periods of time. The form of this time dependence is often logarithmic in time rather than exponential. The reasons for this lie in the distribution of relaxation times which arise typically from distributions of grain volumes or anisotropy constants. Analytical and computational models of slow relaxation in particulate and thin film materials are reviewed by Chantrell et.al. [8]. These processes tend to take place over relatively large energy barriers and typically involve energy barriers of around $25 \mathrm{kT}$. This 
leaves the slow relaxation process relatively insensitive to the value of $f_{0}$. The shortcomings of equation (1) become much more apparent when considering relaxation in short time scales which takes place over relatively small energy barriers. In terms of practical applications, the switching speeds in magnetic recording are now taking place in time scales of ns, which is the region in which equation (1) begins to break down. Clearly there is an important technical imperative for understanding the theory behind magnetic relaxation at very high frequencies. This is an area which is difficult even for a single spin. Of course, most recording media consist of grains which are strongly coupled via both magnetostatic and exchange interactions, leading to a very complex reversal mechanism. The simulation of such systems is an extremely complex problem which is only just beginning to be studied. In this paper we outline the formalism for the theoretical study of thermally assisted magnetisation reversal. We concentrate first on a simple approach for a single isolated spin and then discuss how these ideas can be applied to more complex micromagnetic systems. Calculations of the variation of coercivity with time for a single spin are also presented. Finally we consider the effects of interactions on the detail of the relaxation process.

\section{ANALYTICAL MODELS FOR TIME DEPENDENCE}

We first present a simple analytical model which assists with the interpretation of the form of the time dependence. It also allows the derivation of a simple law relating coercivity to measurement time. In magnetic materials the time-dependence effects arise due to the fact that there is a finite relaxation time $\tau$ required for the magnetisation vector to rotate from one minimum to another. The origin of the energy barrier is a material property and since its magnitude depends on experimental conditions such as magnetic field, these factors govern the probability of thermally activated transitions. In general the time variation of the magnetization of any system can be characterized by a simple differential equation:

$$
\frac{\mathrm{dM}(\mathrm{t})}{\mathrm{dt}}=-\frac{\mathrm{M}(\mathrm{t})-\mathbf{M}_{\mathrm{e}}}{\tau},
$$

where $\mathrm{M}_{\mathrm{e}}=(\mathrm{t}=\infty)$ is the equilibrium magnetization. $\tau$ is the relaxation time given by the Arrhenius-Neel law. In principle the time variation of magnetization can be described by solving equation (2).

For the case of a noninteracting system which contains a distribution of energy barriers the solution of equation (2) gives

$$
M(t)=B+A \int_{0}^{\infty} e^{-t / \tau(y)} f(y) d y
$$

where $B=M(\infty)$ and $A=M(t=0)-M(\infty)$ are timeindependent constants. $f(y)$ is the distribution of energy barriers and $y=E_{b} / E_{b m}$ is the reduced energy barrier relative to the median barrier $E_{b m}$ of the system. $\tau^{-1}(y)=$ $f_{0} \exp (-a y)$ is the inverse of the relaxation time and $a=$ $E_{b m} / k T$. According to equation (3) the time-dependent behaviour is most sensitive to two parameters which characterise the relevant distribution of energy barrier for the system i.e. the average energy barrier of the system and the width of the distribution.

For a wide distribution of energy barriers a $\ln (t)$ law is a good approximation over the range of time examined, i.e. $M \alpha \operatorname{Ln}(t)$. In order to understand the physical interpretation of these results and examine the role of the energy barrier distribution, it is possible to simplify the integral in equation (3) using the critical energy barrier of activation $\left(\mathrm{E}_{\mathrm{c}}\right)$. This critical barrier can be defined in such a way that activation over barriers lower than $E_{c}$ happens so quickly that $\mathrm{e}^{-t / \tau}\left(y<y_{c}\right)$ $\approx 0$. With $\mathrm{e}^{-\mathrm{t} / \tau} \approx 1$ for $\mathrm{y}>\mathrm{y}_{\mathrm{c}}$ equation (3) becomes

$$
\mathrm{M}(\mathrm{t})=\mathrm{B}+\mathrm{A}-\mathrm{A} \int_{0}^{y_{c}(t)} f(y) d y
$$

where $y_{c} \quad E_{c} / E_{b m}$ is the reduced critical barrier for reversal and is given using equation (1) by

$$
\mathrm{y}_{\mathrm{c}}=\frac{1}{\mathrm{a}} \ln \left(\mathrm{tf}_{0}\right) .
$$

According to equation (4) the rate of change of $M(t)$ with $\ln (t)$ is given by

$$
\frac{d M(t)}{d \ln (t)}=-\frac{A}{a} f\left(y_{c}\right) .
$$

This result explains the shapes of any $M(t)$ vs $\ln (t)$ curve since the slope of the $M(t)$ vs $\ln (t)$ curve at any instant is directly linked to the behaviour of the distribution function about the critical barrier above which thermal activation is taking place [10]. In the case of a wide distribution of energy barriers, the variation of $M(t)$ vs $\ln (t)$ is almost linear due to the fact that $f\left(y_{c}\right)$ does not change appreciably about the critical barrier as in the case of a narrow distribution. An analytical expression of equation (4) can be derived by representing the behaviour of $f(y)$ about $y_{c}$ using a series expansion [11][12] 


$$
M(t)=M\left(t_{0}\right)-A \quad \sum_{n=0} f^{n}\left(y_{c}\right)\left[\frac{\Delta \ln (t)}{a}\right]^{n+1},(7)
$$

where

$$
M\left(t_{0}\right)=B+A-A \int_{0}^{y_{c}\left(t_{0}\right)} f(y) d y
$$

and $\Delta \ln (t)=\ln \left(t / t_{0}\right)$ and $t_{0}$ is the initial time of measurement. $f^{n}\left(y_{0}\right)$ is the nth differential of the distribution function evaluated at $y_{c}$. Equation (7) shows that for a constant probability of activation

$$
M(t)=M\left(t_{0}\right)-\frac{A}{a} \Delta \ln (t),
$$

which is the well-known logarithmic time dependence of magnetisation [2].Thus we have two extreme forms of time dependent behaviour; the exponential dependence of a single spin and the logarithmic behaviour of a system with a wide energy barrier distribution. These represent useful comparative means of investigating and characterising relaxation processes.

Finally we derive an important expression for the variation of coercivity with measurement time. This is simply done by noting that from equation (4) the $M=$ 0 state is obtained when precisely half the distribution has switched which, from the definition of the median energy barrier occurs for $y_{c}=1$. This leads to the expression

$$
\mathrm{H}_{\mathrm{c}}=\mathrm{H}_{\mathrm{k}}\left[1-\left\{\operatorname{Ln}\left(\mathrm{tf}_{0}\right) \frac{\mathrm{kT}}{\mathrm{KV}_{\mathrm{m}}}\right\}^{\frac{1}{2}}\right]
$$

where $V_{m}$ is the volume of the particles corresponding to the median diameter. Equation (9) was first derived for a single spin by Sharrock [13].

\section{DESCRIPTION OF THE MODEL}

We have developed a model of the behaviour of a set of interacting particles taking into account the effects of thermal activation. In the absence of thermal perturbations the equation of motion of a single spin is the Landau-Lifschitz equation

$$
\frac{\mathrm{d} \mathbf{M}}{\mathrm{dt}}=\gamma_{0} \mathbf{M} \times \mathbf{H}-\frac{\alpha\left|\gamma_{0}\right|}{\mathrm{M}} \mathbf{M} \times \mathbf{M} \times \mathbf{H}
$$

where $\gamma_{0}$ is the gyromagnetic ratio and $\alpha$ is the damping parameter. The properties of the deterministic equation (10) are well known. In the absence of damping the first term leads to an infinite precession. The effect of the damping term is to produce a damped precessional motion which eventually leads to an equilibrium position in which the magnetisation is collinear with the local magnetic field. Following Brown, we can introduce the effects of thermal activation by adding a random field term $h(t)$ to the Landau-Lifschitz equation. Taking the random field in Cartesian co-ordinates, ie.

$$
h(t)=h_{1}(t) \hat{\mathbf{x}}+h_{2}(t) \hat{\mathbf{y}}+h_{3}(t) \hat{z}
$$

the components of the random field must have the following statistical properties

$$
\begin{aligned}
& \left\langle h_{i}(t)\right\rangle=0 \\
& \left\langle h_{i}(t) h_{i j}\left(t+t^{\prime}\right)\right\rangle=\mu \delta_{i j} \delta\left(t^{\prime}\right)
\end{aligned}
$$

where the 2 nd of equation 4 is a manifestation of the fluctuation-dissipation theorem, and asserts that

i. different components are uncorrelated

ii. for $\mathrm{i}=\mathrm{j}$ the fields are uncorrelated certainly for times $\mathrm{t}^{\prime}>10^{-13} \mathrm{~s}$, which is the typica correlation time for the thermal perturbations.

With the addition of $\mathbf{h}(t)$ the Landau-Lifschitz equation is converted to the Langevin equation of the problem, which can be solved numerically or under certain circumstances analytically.

It is straightforward to show under the assumption that the random process results from the superimposed effects of a large number of independent random events, then by virtue of the central limit theorem the statistical distribution of the random variable is Gaussian with a variance of $\mu$. Standard numerical techniques exist for picking a normal deviate with a specified variance, and using these techniques it is possible to randomly choose the fluctuating field using equation (3). Consequently it is possible to solve the stochastic equation of motion under the influence of thermal effects. In order to determine the value of $\mu$ we consider first the approach pioneered by Brown, which involves transforming the Langevin equation into the Fokker-Planck (F-P) equation of the problem.

Following the method of Brown of transforming the Langevin equation into the FokkerPlanck equation and imposing the Boltzmann distribution on the equilibrium probability density it can be shown that 


$$
\mu=\frac{2 k T}{V} \frac{1}{M|\gamma|} \frac{\alpha}{\left(1+\alpha^{2}\right)},
$$

where $\mathrm{V}$ is the particle volume. It has been shown [14] that the introduction of thermal activation gives rise to a random walk toward equilibrium, followed by a random precession about the local field direction. Macroscopically this results in magnetisation fluctuations [15]

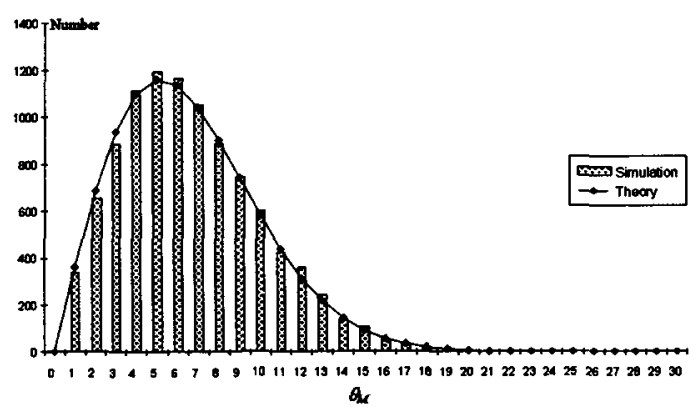

Figure 1: Distribution of magnetisation orientations as a function of $\theta_{M}$.

As shown in figure 1 the predicted orientational distribution for a set of non-interacting particles with easy axes aligned with the applied field obeys the Boltzmann distribution function

$$
\mathrm{W}=\mathrm{A}_{0} \exp \left[\frac{-\mathrm{E}(\theta)}{\mathrm{kT}}\right],
$$

where $\mathrm{W}$ is the probability density. In terms of micromagnetic simulations we note that this is already an important factor. Standard micromagnetic simulations proceed by determining equilibrium states, with magnetisation reversal taking place when the local energy minimum vanishes. However, at this point there is zero torque on the spins. Thermal fluctuations break the symmetry and ensure a torque on the moment at all times since the moment is no longer parallel to the local field. Thus they provide, in essence, a physical probe of the stability of the equilibrium state.

In this paper we present a number of simulations of non-interacting systems in order to investigate nonexponential behaviour for single spins at very short time scales and the effects of a particle size distribution. In addition we have used the model to study the effects of interparticle interactions. The model assumes a cubic computational cell, extended in 3D using periodic boundary conditions. The particles are assigned a diameter from a lognormal size distribution with specified median diameter and standard deviation. The anisotropy is assumed uniaxial, and the orientational distribution can be specified from completely random to perfectly orientated. In the simulations presented here perfect alignment in the field direction was assumed for clarity of interpretation. Particles are placed at random in the computational cell, rejecting overlapping placements. In order to achieve high packing densities the particles were placed in the cell in order of decreasing particle size, allowing smaller particles to fill interstitial sites.

Interactions were introduced by adding to the applied field an interaction field

$$
\mathrm{H}_{\mathrm{i}}=\sum_{i=j} \frac{3\left(\mu_{i}, \hat{r}_{i j}\right) \hat{r}_{i j}-\mu_{j}}{\left(d_{i j}\right)^{3}},
$$

where the summation is taken over a cut-off radius. Clearly the total local field will vary in magnitude and direction due to interaction effects. Such fluctuations might be expected to have an important bearing on the relaxation behaviour of the material, and especially the form of the high frequency properties.

\section{RESULTS}

We first present results for a non-interacting system in order to study the detailed form of the relaxation and also the effect of a particle size distribution. The system considered contains 1000 noninteracting ferrite particles, the first having a uniform particle size ( $24 \mathrm{~nm}$ in diameter) and the second having a lognormal size distribution with median diameter 24 $\mathrm{nm}$ and standard deviation 0.2 . The particles have uniaxial anisotropy with easy axes aligned in the direction of the applied field. We study the fast relaxation behaviour by firstly saturating the sample and then applying a large negative field. The behaviour of the individual spin is calculated using equation (10). We then monitor the number of particles having their original magnetisation at the end of a pulse of given duration t. Essentially this is the remanent magnetisation after the pulse.

Figure 2 compares the relaxation of the monodisperse and distributed systems. We note firstly that the relaxation for a single volume is nonexponential. This should perhaps not be considered surprising, since the exponential law arises for the large energy barrier case, where it is reasonable to assume a well-defined 2-state system. Generally speaking, analytical theories proceed by transforming the Langevin equation into the Fokker-Planck equation of the problem, essentially by considering a large ensemble 


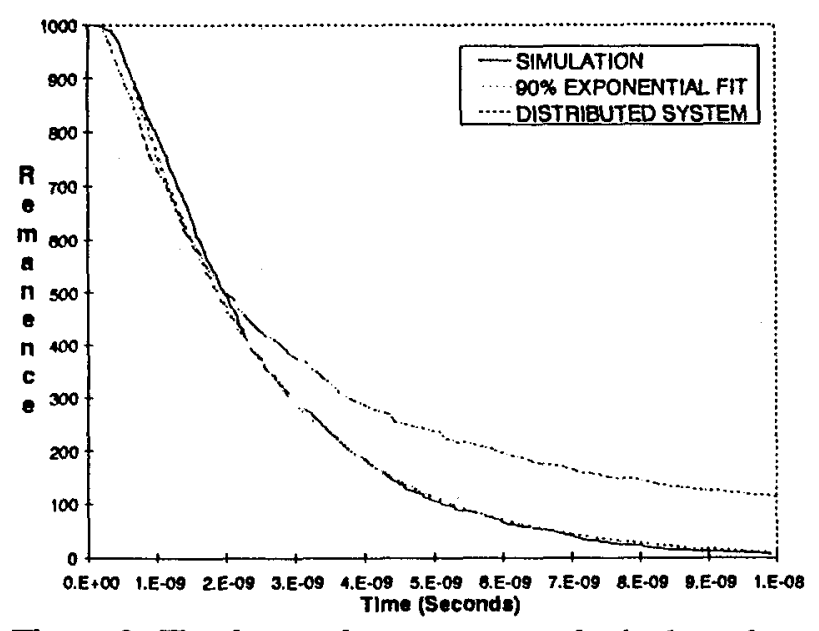

Figure 2: The decay of magnetisation for both uniform and distributed volumes.

of particles characterised by a probability density $W(\theta, \phi, t)$ on the unit sphere. Series expansion of $W$ produces an eigenvalue problem which can be solved numerically and also analytically in some limiting cases (for example large energy barriers). It is generally assumed that the characteristic relaxation time is associated with the lowest eigenvalue. Of course, for small energy barriers one expects the exponential law to break down. Brown for example [16] gives an entirely different form for the relaxation for small energy barriers. Unfortunately this asymptotic expansion is valid only for a small field in addition to the requirement of a small energy barrier. It seems reasonable to suggest that the non-exponential behaviour shown by our simulations reflects the breakdown of the high energy barrier approximation and also possibly the increasing importance of higher eigenvalues. The deviation from exponential behaviour occurs at the shortest timescales, where the relaxation is initially very flat. Over longer timescales the relaxation is more closely exponential. We demonstrate this by fitting an exponential law to the last $90 \%$ of the curve which, as shown in figure 2 reproduces the form of the computational results rather well.

Figure 2 also shows the relaxation for the distributed system. The relaxation is more strikingly non-exponential, as expected from the spread of relaxation times. After the initial slow decay similar to that of the monodisperse system, the relaxation time distribution is reflected in the initially faster decay which slows down significantly at longer times. This behaviour is close to logarithmic over 1 or 2 decades, consistent with the effects of a spread of relaxation times as expressed in equation (8).

The introduction of magnetostatic interparticle interactions is found to give rise to a more rapid variation, as shown in figure 3 , consistent with the anticipated

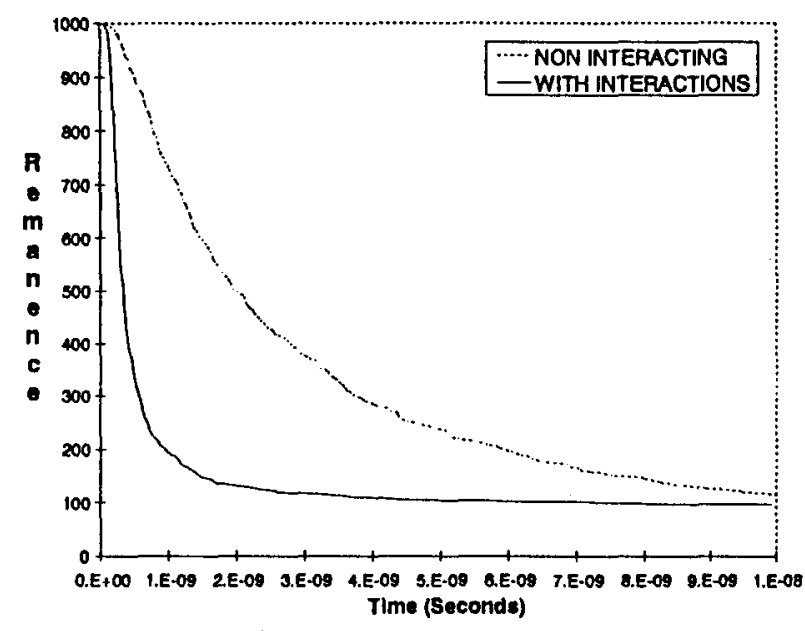

Figure 3: The decay of magnetisation for interacting and non-interacting systems with distributed volume.

reduction in the coercive force.

In comparison with the non-interacting case the relaxation is much more rapid initially. This can be ascribed to the demagnetising effect of the particle interactions, which tend to lead to flux closure configurations thereby stabilising the demagnetised state. At longer times, however, the relaxation slows down dramatically, eventually tending toward the noninteracting case for times approaching $10^{-8} \mathrm{~s}$. A useful comparison between the data can be made by scaling the data with respect to a relaxation time $\tau_{1 / 2}$ defined as the time to fall to $1 / \mathrm{e}$ of the original population. Plots of remanence vs $t / \tau_{1 / 2}$ for the interacting and noninteracting systems are given in figure 4 . On this scale it can be seen that the effect of interactions is relatively to slow down the relaxation at short and long time scales. This may perhaps be associated with a broadening of the energy barrier distribution due to the dispersion in local interaction fields, which we recall is distributed in both magnitude and direction.

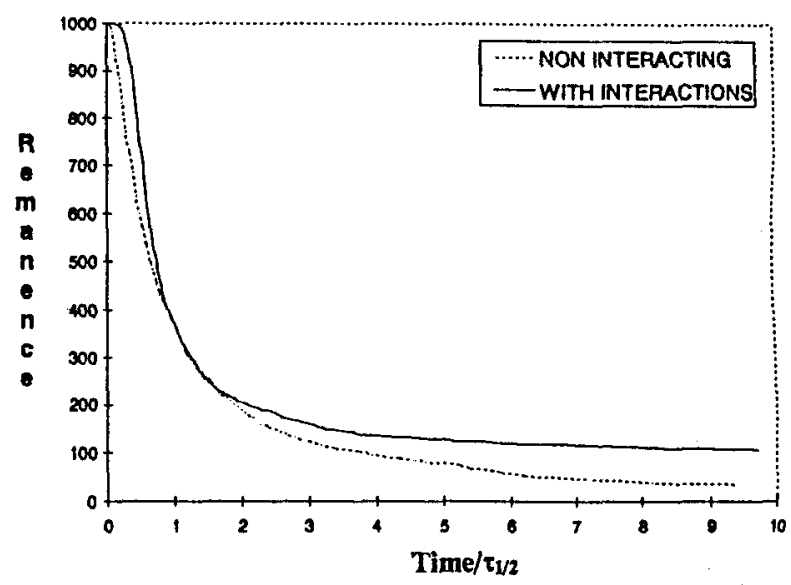

Figure 4: The decay of magnetisation for interacting and non-interacting systems scaled to $\tau_{1 / 2}$. 
Clearly the relaxational behaviour of both the interacting and non-interacting systems is strongly nonexponential. We therefore compare the data with the other limiting case discussed earlier of logarithmic time dependence. A plot of remanence vs $\ln t$ is shown in figure 5 for the last stages of relaxation of the interacting system. As might be expected the relaxation is not precisely logarithmic. We note, however, a distinct similarity between these results and the Montecarlo calculations of Lyberatos et. at. [17] on thin films with perpendicular anisotropy. In this case the rapid relaxation is driven by a strong demagnetising field which leads to a slowing down as the magnetisation tends to zero. In the present data the large applied field causes the initial rapid demagnetisation, with the slowing down at long times arising from those particles with large energy barriers due to a combination of the larger particle volume and the effects of the interaction fields.

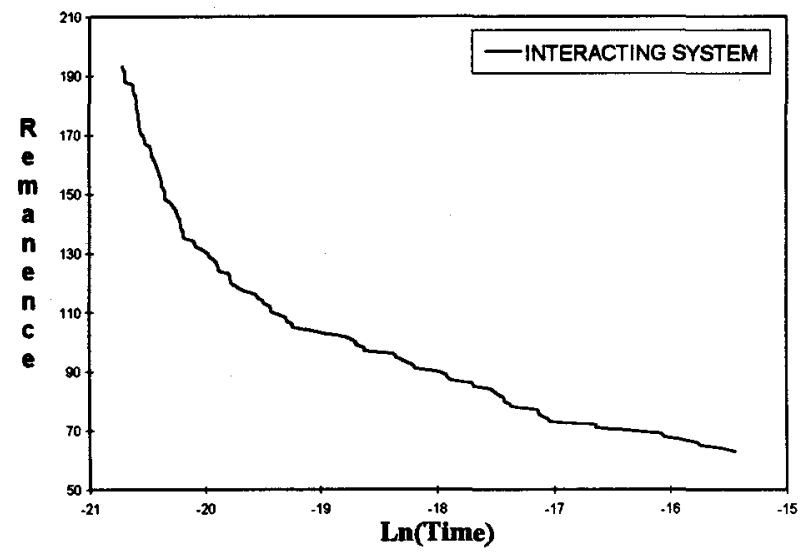

Figure 5: The decay of magnetisation for the interacting system against a log time scale.

The long time scale behaviour shown in figure 5 is more closely logarithmic than exponential, as would be expected from a system with a distribution of energy barriers arising from the dispersion of particle size and local field. However, for a non-interacting system with such a wide distribution of particle size the variation might be expected to be closely logarithmic. The curvature in the plot may well result from the effects of the time varying magnetic state, in an analogous manner to the behaviour observed in [17]. In the previous work [17] it could be concluded that the form of the time dependence was dominated by the very strong local demagnetising field. In our current simulations there is no such easily identifiable magnetisation dependent contribution to the local field. Our current results suggest that subtle changes in the magnetic state can make a similar contribution for example via local fluctuations in the interaction field as the system moves between local minima on the energy surface.

Finally we turn to simulations of the coercivity at high frequency. Here we consider a monodisperse, non-interacting system of particles with easy axes aligned with the applied field. The simulation is carried out by applying a reverse field to an initially saturated assembly and then calculating the time taken for $50 \%$ of the particles to reverse. Typical results are shown in figure 6 which gives $\mathrm{H}_{\mathrm{k}} \approx 164 \mathrm{kAM}^{-1}$ and volume $\mathrm{V}=$ $7.5 \times 10^{-24} \mathrm{~m}^{3}$, at a temperature $\mathrm{T}=300 \mathrm{~K}$. The damping constant $\alpha$ was taken to be 0.1 . It can be seen that the coercivity increases to a value greater than $\mathrm{H}_{k}$. This is due to the fact that if the field is applied for too short a time the spin may not rotate over the energy barrier, in which case when the field is switched off it will simply rotate back to its original energy minimum and not make the transition. Thus at short time scales, fields considerably larger than $H_{k}$ are required for magnetisation reversal. Clearly this rapid increase of coercivity at short time scales represents an important limitation in terms of recording speed. In figure 6 we make a comparison with the Arrhenius-Neel law prediction (equation 9). For times greater than $10^{-8} \mathrm{~s}$ there is good agreement between the simulation and the analytical results, bearing in mind the assumed constancy of $f_{0}$. The divergence at short timescales is directly attributable to the breakdown of the ArrheniusNeel law at low energy barriers. As mentioned previously, the Arrhenius-Neel law is applicable only to well-defined 2-state systems and also makes the crude assumption of the constancy of the pre-exponential factor $f_{0}$. As $T$ decreases ( and $H_{r}$ increases) $E_{b} / k T$ tends to unity, thus the assumption of the exponential relaxation is not justified. In addition, the field dependence of $f_{0}$ predicted by Brown is a further complication. What is required is a new analytical approach valid for small energy barriers and high fields.

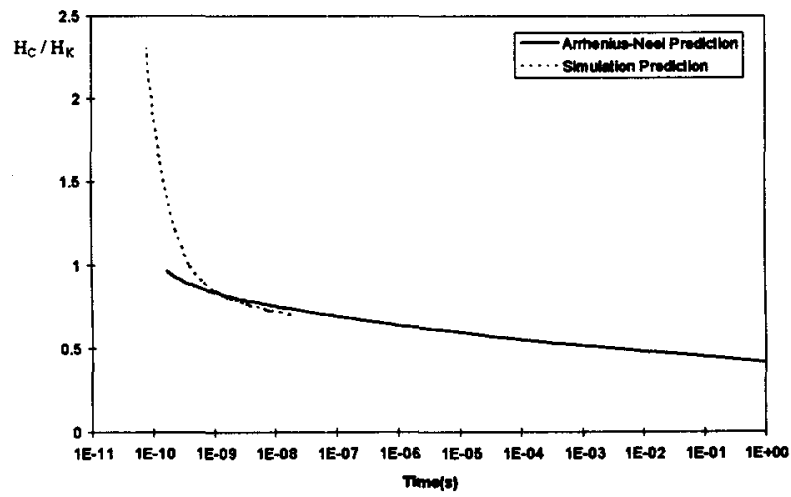

Figure 6: The variation of remanence coercivity with switching time. 


\section{ACKNOWLEDGEMENTS}

We are grateful for interesting discussions with Dr I Klik and Dr H-J Richter. The financial support of EPSRC is gratefully acknowledged. J D Hannay also wishes to thank Seagate (Northern Ireland) for their support of a CASE studentship.

\section{REFERENCES}

[1] A Ewing, Phil. Trans. R. Soc., 176, 569 (1885)

[2] R Street and J C Wooley, Proc. Phys. Soc., A 62, 562 1949)

[3] E C Stoner and E P Wohlfarth, Phil. Trans. R Soc., A 240, 599 (1948)

[4] L Neel, Ann. Geophys., 5, 99 (1949)

[5] W F Brown, Phys. Rev., 130,1677 (1963)

[6] A Aharoni, Phys. Rev., A 135, 447 (1964)

[7] W T Coffey, P J Cregg and Y P Kalmykov, Adv. Chem. Phys. 83, 263 (1993)

[8] R W Chantrell, A Lyberatos, $\mathrm{M}$ El-Hilo and $\mathrm{K}$ O'Grady, J. Appl. Phys. 766407 (1994)

[9] L Neel, J. Phys. Rad., 12339 (1951)

[10] M El-Hilo, O'Grady and R W Chantrell, J. Magn. Magn. Mater. 109 L164 (1992)

[11] M El-Hilo, K O'Grady and R W Chantrell. J. Magn. Magn. Mater. 12330 (1993)

[12] K O'Grady, M El-Hilo and R W Chantrell, J. Appl. Phys. 76, 10 Pt.2 (1994)

[13] M P Sharrock, IEEE Trans. Mag., 26, 193 (1990)

[14] R W Chantrell, J D Hannay, M Wongsam and A Lyberatos. IEEE Trans. Mag. (in press)

[15] $Y$ Nakatani, $Y$ Uesaka, $N$ Hayashi and $H$ Fukushima, J. Magn. Magn. Mater. 168, 347 (1997)

[16] W F Brown Jr., IEEE Trans. Mag. 151196 (1979)

[17] A Lyberatos, R W Chantrell and A Hoare, IEEE Trans. Mag. 26, 222 (1990) 